\title{
Psychosocial Adjustment in Women with Schizophrenia using the Tamil Version of B-WISE
}

\author{
Hema Tharoor $^{1} \cdot$ Vidhya Mohandoss $^{1} \cdot$ Sindhu Maran $^{1} \cdot$ Subhashini Gopal $^{1}$
}

Received: 2 May 2015/ Accepted: 25 June 2015/Published online: 15 July 2015

(C) Springer India Pvt. Ltd. 2015

\begin{abstract}
Obesity due to antipsychotic induced weight gain is a significant problem in patients with schizophrenia. An instrument to evaluate body image and self-esteem (BWISE) related to weight has recently been developed. To evaluate psychosocial adjustment based on body image and self esteem using B-WISE in Schizophrenia. In a crosssectional case control design, 82 female patients attending Schizophrenia Research Foundation services were recruited. The translated Tamil version of the B-WISE was administered by two raters independently within a 3 week interval to establish test-retest reliability. Scores on B-WISE categories differed significantly between cases and controls $(\mathrm{p}=0.034)$ but majority reported good psychosocial adjustment. Test restest reliability of the tamil version of the B-WISE was found to be good with kappa value 0.78 . B-WISE could be a useful screening instrument to evaluate the psychosocial consequences associated with weight gain in patients with schizophrenia.
\end{abstract}

Keywords Obesity · Psychosocial adjustment · B-WISE · Schizophrenia

Hema Tharoor

hematharoor@scarfindia.org

1 Schizophrenia Research Foundation (India), R/7A, North Main Road, Anna Nagar West Extension, Chennai 600101, India

\section{Introduction}

Body image may be conceptualized as a multidimensional construct that represents how individuals think, feel, and behave with regard to their own physical attributes [1]. Medications used to treat schizophrenia are often associated with weight gain. Consequences of antipsychotic drug induced weight gain include decreased self-esteem, reduced quality of life, and poor adherence with medications. Recently, the body weight, image and self-esteem (B-WISE) instrument has been used to examine body image in patients treated with antipsychotic medication [2]. In serious mental illness (SMI), the B-WISE is believed to capture the psychosocial impact of antipsychotic-related weight gain [3]. Two large studies conducted in the West have shown adequate psychometric properties for the B-WISE in persons with SMI [3, 4]. Patients with higher BMIs report more negative body image perceptions than those patients with lower BMIs $[2,5,6]$. Patients experiencing a recent weight gain had lower self-esteem and poorer psychosocial adaptation [3, 7]. The Tamil version of the B-WISE is being tested for validity and reliability in a larger study at Schizophrenia Research Foundation (SCARF). Keeping this in mind, this study was conceived to find out the psychosocial impact of body image and self esteem in patients with chronic schizophrenia using the B-WISE and establish test-retest reliability of the tool.

\section{Aims and Objectives}

(1) Compare BMI \& Psychosocial adjustment using Tamil version of B-WISE instrument in female patients with Schizophrenia vs healthy controls. 
(2) Establish inter-rater reliability of the Tamil version of the B-WISE questionnaire.

\section{Materials and Methods}

Study design Case control study.

Study period 30 days (May 2014)

Sampling Serial sampling

Sample size Total 82 (Cases-41, Controls-41)

\section{Target Population}

\section{Cases-Inclusion Criteria}

(1) All female patients ( $>18$ years) diagnosed with schizophrenia (ICD-10)

(2) Patients who give consent for the study

(3) Patients on stable dose of medications

(4) Stable doses defined as no change in medications in the last 3 months

\section{Exclusion Criteria}

(1) Patients who are uncooperative due to the severity of the illness or reporting of somatic delusions

(2) Not willing to participate in the study

(3) Patients with mental retardation, head injury squeal, seizures

(4) Not on stable dose of medications in the last 90 days

\section{Controls-Inclusion Criteria}

(1) Weight matched normal females who give consent for the study

(2) Women scoring $<15$ on the General Health Questionnaire

\section{Exclusion Criteria}

Not willing to participate.

\section{Tools}

\section{Proforma}

A semi structured proforma was designed to collect information regarding the socio demographic data, Body mass index (BMI) and illness related variables including treatment. BMI will be calculated and patients will be categorised as underweight (<18.49), normal (18.5-24.9), overweight (25-29.9) or obese $(>29.9)$ based on WHO guidelines.

\section{Body Weight, Image and Self Esteem Questionnaire (B-WISE) [2]}

The B-WISE is a 12-item self-report questionnaire, and items are drafted as first person statements describing a subject's personal appraisal of the changes in body weight and issues related to psychosocial adjustment in the preceding 2 weeks. Answers are chosen from a three-point frequency based Likert scale (i.e., never, sometimes and all the time). The answers are assigned a rating of 1,2 or 3 , with potential total scores ranging between 12 and 36, higher scores indicative of better adjustment. Psychometric properties show high internal consistency (Cronbach's alpha 0.79) and fair split half reliability (Spearman-Brown coefficient of 0.76). Test-retest reliability coefficient of $0.81(p<0.001)$. Tamil version of the B-WISE was used in this study. 2 independent persons did translation and back-translation of the Tamil version of the instrument. The kappa value was found to be 0.785 showing that the test-retest reliability of the tamil version B-WISE questionnaire is good.

\section{General Health Questionnaire (GHQ) [8]}

GHQ is a 12-item self-reporting questionnaire and items are drafted as first person statements. This scale intends to detect psychiatric disorders in community setting. Answers are chosen in a 3 point frequency based Likert scale ranging from 0 to 3 . Scores are calculated by summing up all items on the scale from 0 to 12 . Scores range from 0 to 36. High scores are indicative of severe psychological distress. The reliability coefficient has ranged from 0.78 to 0.97 in various studies.

\section{Ethical Clearance}

Institutional Ethics Committee clearance was obtained prior to the commencement of the study.

\section{Procedure}

In a naturalistic cross-sectional study all women admitted to SCARF residential centres were screened during the study period. A total of 75 eligible patients were approached and forty one consented to participate in the study. Patients signed a written informed consent and those fulfilling the inclusion and exclusion criteria were recruited as cases. A 
total of 80 controls were approached so that 41 healthy participants fulfilling the study criteria were recruited. Healthy age and weight matched women who consented to participate and fulfilling the inclusion and exclusion criteria for controls were screened using the GHQ to establish normality. Tamil version of the B-WISE questionnaire was administered in a single sitting. The same questionnaire was administered by a different rater within an interval of 3 weeks to establish inter-rater reliability on the same patient. The socio demographic data, duration of the illness, type of medications were collected from the medial records. Medications received were either single or more than one antipsychotic. The BMI was calculated prior to the administration of the B-WISE questionnaire. Body mass index was calculated and patients were categorised as underweight, normal, overweight or obese based on WHO guidelines.

\section{Data Analysis}

Analysis of data was done using the Statistical package for Social sciences (SPSS) 16 version [9].

\section{Results}

A total of 82 patients (41 cases and 41 controls) were recruited in the study as depicted in Table 1. Among the subjects $48.8 \%$ of cases were single and majority had duration of illness more than 10 years $(80 \%)$ and were receiving polypharmacy $(95.2 \%)$. An equal proportion of cases $(48.8 \%)$ and controls $(46.3 \%)$ were found to be in the overweight category of BMI. Majority of cases and controls were found to be in the mild category of cumulative B-WISE scores indicating good psychosocial adjustment. Table 2 shows the comparison of B-WISE scores and was found to be statistically significant. Correlational analysis was done between duration of illness, BMI and treatment with B-WISE categories was not significant. Graph 1 depicts that BWISE comparisons in the overweight and obese categories of both groups were not significant. BWISE questionnaire description and ratings for cases and controls are listed in Table 2. Comparison of the two items Q3-“Going out to enjoy myself more often” and Q10- "I believe that excess weight is not good for my general health" were significantly more in controls than in cases.

\section{Discussion}

Body image is a heterogeneous construct that is influenced by a variety of biological, psychological, and social factors. The B-WISE considers one aspect of this model-body weight. Examination of the participant demographic characteristics suggests that our sample was similar in sex and BMI as the two previous B-WISE studies [2, 3]. In agreement to previous research B-WISE exhibited good

Table 1 Sample description

\begin{tabular}{|c|c|c|c|}
\hline Variables & Cases $\mathrm{N}_{1}=41(\%)$ & Controls $\mathrm{N}_{2}=41(\%)$ & Total $\mathrm{N}=82(\%)$ \\
\hline Mean age (SD) & $49.2 \pm 10.1$ & $36.5 \pm 11.1$ & $42.9 \pm 12.3$ \\
\hline \multicolumn{4}{|l|}{ Marital status } \\
\hline Single & $20(48.8)$ & $15(36.6)$ & $35(42.7)$ \\
\hline Married & $7(17.1)$ & $26(63.4)$ & $33(40.2)$ \\
\hline \multicolumn{4}{|l|}{ Duration of illness } \\
\hline$<5$ years & $4(10)$ & N/A & \\
\hline $5-10$ years & $4(10)$ & & \\
\hline$>10$ years & $33(80)$ & & \\
\hline \multicolumn{4}{|l|}{ Medications type } \\
\hline Monotherapy & $2(4.8)$ & N/A & \\
\hline More than one antipsychotics & $39(95.2)$ & & \\
\hline \multicolumn{4}{|l|}{ BMI } \\
\hline Underweight & $1(2.4)$ & $3(7.3)$ & $4(4.9)$ \\
\hline Normal & $8(19.5)$ & $11(26.8)$ & $19(23.2)$ \\
\hline Over weight & $20(48.8)$ & $19(46.3)$ & 39 (47.6) \\
\hline Obesity & $12(29.3)$ & $8(19.5)$ & $20(24.4)$ \\
\hline \multicolumn{4}{|l|}{ BWISE } \\
\hline Mild & $23(56.1)$ & $31(75.6)$ & $54(65.9)$ \\
\hline Moderate & $13(31.7)$ & $10(24.4)$ & $23(28.0)$ \\
\hline Severe & $5(12.2)$ & 0 & $5(6.1)$ \\
\hline
\end{tabular}


Table 2 Comparison of B-WISE category between cases and controls

\begin{tabular}{lcll}
\hline BWISE category $\mathrm{N}(\%)$ & Cases $(\mathrm{N}=41)$ & Control $(\mathrm{N}=41)$ & Chi square \\
\hline Mild & $23(56.1)$ & $31(75.6)$ & $.037^{*}$ \\
Moderate & $13(31.7)$ & $10(24.4)$ & \\
Severe & $5(12.2)$ & 0 & \\
\hline
\end{tabular}

$* \mathrm{p}<0.05$

reliability (kappa value $=0.78$ ) [2]. Majority of patients had scored in the mild category indicating good psychosocial adjustment to medication induced weight gain. This is an expected finding in our study as majority of our patients had longer illness duration and were on antipsychotic treatment for prolonged period of time. In previous studies patients with higher BMIs report more negative body image perceptions than those patients with lower BMIs. However participants in our study were predominantly in the overweight and obese group and did not have negative perceptions. This could be explained by the chronicity of illness, drugs received, lack of physical activity participation and in-patient setting. Surprisingly, majority of healthy obese/overweight controls showed a lack of negative perception and enjoyed going out more often. Controls were younger and comparatively higher educated than the patients could explain this difference. Body image is potentially influenced by the sociocultural and background of the study participants. Both groups and significantly more controls believed that excess weight is not good for general health. This is an important finding because patients are at a risk of metabolic syndrome due to antipsychotics and need awareness about weight management and life style modification. Patients and controls were equally distributed for age \& weight so given the smaller sample size and potential differences in two items of the B-WISE instrument the validity, internal consistency and cultural sensitivity of this tool in the local language is warranted. Content and construct validity of the items in a larger sample may fully capture the concept of body image in persons with Schizophrenia.

\section{Limitations}

A prospective study in drug naïve patients to assess antipsychotic induced weight gain at different intervals could give us a better understanding of the psychosocial impact of B-WISE. A cross-sectional study design may not truly assess the psychosocial impact due to antipsychotic induced weight gain because multiple factors such as years of antipsychotic intake, age, sedentary life style, metabolic syndrome and other co-morbid medical illness need to be accounted in chronic Schizophrenia.

\section{Conclusions}

Poor psychosocial adjustment due to drug induced weight gain can have major implications on treatment adherence, self-esteem and quality of life. The Tamil version of the B-WISE could be a preliminary tool to assess psychosocial adjustment due to drug induced weight gain in patients with Schizophrenia.

\section{Compliance with Ethical Standards}

Conflict of interest The authors declare that they have no conflict of interest.

\section{References}

1. Muth JL, Cash TF. Body-image attitudes: what difference does gender make? J Appl Soc Psychol. 1997;27:1438-52.

2. Awad GA, Voruganti LNP. Body weight, image and self esteem evaluation questionnaire: development and validation of a new scale. Schizophr Res. 2004;70:63-7.

3. De Hert M, Peuskens B, Van Winkel R, Kalnicka D, Hanssens L, Van Eyck D, Wyckaert S, Peuskens J. Body weight and selfesteem in patients with schizophrenia evaluated with B-WISE. Schizophr Res. 2006;88:222-6.

4. AlHalabi S, Garcia Portilla MP, Saiz PA, Fonseca E, Bobes Bascaran MT, Galvan G, et al. Psychometric properties of the Spanish version of the body weight, image and self-esteem evaluation questionnaire in patients with severe mental disorders. Compr Psychiatr. 2012;53:1237-42.

5. Strassing M, Brar JS, Ganguli R. Self-reported body weight perception and dieting practices in community-dwelling patients with schizophrenia. Schizophr Res. 2005;75:425-32.

6. WHO Expert Consultation. Appropriate body-mass index for Asian populations and its implications for policy and intervention strategies. Lancet. 2004;363:157-63.

7. Weiden PJ, Mackell J, McDonnell DD. Obesity as a risk factor for antipsychotic noncompliance. Schizophr Res. 2004;66:51-7.

8. Goldberg D. General health questionnaire (GHQ12). Windsor: NFER-Nelson; 1992.

9. SPSS version 16 (SPSS Inc., Chicago, Illinois. 